\title{
The spectral variability of a sample of WC 9 stars on time-scales of days to weeks
}

\author{
S. Desforges, ${ }^{1}$ N. St-Louis, ${ }^{1 \star}$ A.-N. Chené, ${ }^{2}$ A. de la Chevrotière ${ }^{1}$ \\ and V. Hénault-Brunet ${ }^{3}$ \\ ${ }^{1}$ Département de Physique, Université de Montréal, C.P. 6128, Succ. Centre-Ville, Montréal, Québec H3C 3J7, Canada \\ ${ }^{2}$ Gemini Observatory, Northern Operations Center, 670 North A'ohoku Place, Hilo, HI 96720, USA \\ ${ }^{3}$ Department of Astrophysics/IMAPP, Radboud University, PO Box 9010, NL-6500 GL Nijmegen, The Netherland
}

Accepted 2016 October 25. Received 2016 October 25; in original form 2016 August 25

\begin{abstract}
We present the results of a spectroscopic monitoring campaign of nine presumably single Wolf-Rayet (WR) stars, eight of type WC 9 and one WC 8d. We characterize their variability and search for clues to the mechanism responsible for the formation of dust in their wind. For seven out of eight WC 9s, we find a large-scale line-flux variability level of $\sigma>5-8$ per cent. The only WC $8 \mathrm{~d}$ star is variable at a level more comparable with those associated with wind clumping, $\sigma=2.2$ per cent. The changes take place on a time-scale of days but in many cases, observing over longer time spans resulted in higher line-flux variability levels. The width of the substructures ranges from $\sim 150$ to $300 \mathrm{~km} \mathrm{~s}^{-1}$, with the widest structures corresponding to stars with the highest variability amplitude. We searched for periodicities in integrated line quantities for $\mathrm{C}_{\text {III }} \lambda 5696$. Radial velocity changes are typically $\sim 20 \mathrm{~km} \mathrm{~s}^{-1}$ but never exceed $40 \mathrm{~km} \mathrm{~s}^{-1}$ and are anticorrelated with the skewness of the line, strongly suggesting that they do not correspond to a real movement of the star. No periodicity was found in these integrated quantities, except for WR 103. Therefore, a wind-wind collision in a close binary does not seem to be responsible for the short-term variability. We cannot, however, exclude that these stars are intermediate- to long-period binaries. We estimate that for periods up to a few years, the shock-cone resulting from wind collisions would be non-adiabatic and thus unstable. We suggest that this represents a viable mechanism to explain the spectroscopic variability.
\end{abstract}

Key words: techniques: spectroscopic-stars: winds, outflows-stars: Wolf-Rayet.

\section{INTRODUCTION}

Amongst Wolf-Rayet (WR) stars, those of the WC 9 subtypes are the coolest and least luminous of the WC sequence. The terminal velocity of their wind is generally smaller than that of earlier subtypes and of those of the WN sequence. Their most striking characteristic is that a large fraction of WC 9 stars display in their spectrum an excess of thermal emission in the near-infrared, which has been associated with the presence in the wind of carbon-based dust, most likely amorphous grains (Williams, van der Hucht \& The 1987). There are some exceptions; for example, it was found by Williams \& van der Hucht (2000) that the three stars WR 81 , WR 88 and WR 92, for which no evidence for dust has been found, are characterized by weaker oxygen lines and stronger He II lines and therefore a lower $\mathrm{O} / \mathrm{He}$ abundance ratio. If they are indeed oxygen deficient, the absence of $\mathrm{CO}$ (a precursor in the grain formation chain; Cherchneff et al. 2000) would inhibit the dust formation process. However, more recently, Williams, Crowther \& van der Hucht (2015) suggested that it is a higher carbon abundance that is key in allowing WC stars to make dust rather than a deficit of oxygen, preventing some WC-late stars of producing it.

The physical processes at the origin of dust formation in the harsh environment of a WR wind are still unclear. According to current theories, in an isotropic wind, the grains must be formed as far as $\sim 100$ au from the photosphere, otherwise the strong ultraviolet (UV) radiation from the star would destroy the neutral carbon grains by sublimation (Williams \& van der Hucht 2000). However, in such winds, the densities at these distances are several orders of magnitude too low to allow the processes leading to grain condensation to occur (Cherchneff et al. 2000). Nevertheless, a non-uniform wind could provide regions where the density is high enough to allow dust formation through self-shielding.

One way to produce such regions of higher densities is in colliding-wind binaries (CWBs). Dust formation has indeed been observed in many long-period (years) $\mathrm{WC}+\mathrm{O}$ systems. Some produce dust only at periastron where the collision of the two stellar winds is strong enough to produce shocks that compress the gas to 
densities up to a factor $\sim 10^{3}$ (Usov 1991). These systems are known as 'episodic' or 'periodic' dust makers. WR 140 (WC 7+O5.5) is the archetype of such a colliding-wind system, making dust for about 3-4 months at regular intervals of $7.94 \mathrm{yr}$ (e.g. Williams 2011). Other CWBs such as WR 104 (WC 9+O), famous for its rotating 'pinwheel' pattern of infrared emission (Tuthill, Monnier \& Danchi 1999), have a circular orbit and produce dust on a continuous basis. These are known as 'persistent' dust makers. There is however no conclusive evidence suggesting that all the persistent WC 9 dust makers are part of a binary system with colliding winds. Indeed, Williams \& van der Hucht (2000) obtained high signal-tonoise ratio $(\mathrm{S} / \mathrm{N})$ spectra to search for absorption lines attributable to hot companions in the spectra of $10 \mathrm{WC} 9$ stars and only found evidence for them in two, WR 104 and WR 69. ${ }^{1}$ Note also that some WC 9 stars produce dust persistently but at a variable level. This is the case for WR 98a (Monnier, Tuthill \& Danchi 1999) and WR 70 (Williams et al. 2013), both confirmed binaries.

Observations have clearly shown that the winds of WR stars are highly clumped (e.g. Moffat et al. 1988; Lépine \& Moffat 1999). Hydrodynamical simulations of CWBs with clumpy winds by Pittard (2007) require a density ratio between the clumps and interclump wind of at least 10 to correctly reproduce the observed mass-loss rates. Given that episodic CWBs produce dust only at periastron passage, it is rather unlikely that clumps are dense enough to allow dust formation to occur, otherwise it would be observed continuously albeit at a lower level.

It is also possible for the wind of a single massive star to harbour large-scale zones of compressed gas. Hydrodynamical simulations by Cranmer \& Owocki (1996) have shown that corotating interaction regions (CIRs) in the wind of $\mathrm{O}$ and WR stars will form by the combined effect of a perturbation at the base of the wind and the rotation of the star. This arises when low-density, high-speed unperturbed wind collides with a slower but denser wind zone. The latter wind component is the response of a localized enhancement of the radiative force caused by a bright spot at the hydrostatic radius. Because of the star's rotation, CIRs appear like spiral-shaped structures that are wrapped around the star. In principle, it is possible to determine if a star has one or more CIRs in its wind by studying the excess emission subpeaks superposed on its emission lines. These line profile variation (LPV) patterns are very different from those caused by the clumping of the wind for two reasons: first, they move periodically (i.e. Morel, St-Louis \& Marchenko 1997) and second, the subpeaks on the emission lines are broader (Lépine $\&$ Moffat 1999) and move from one side of the profile to the other crossing the centre rather than from line centre to line edge as subpeaks caused by clumps in the wind do. So far, only three WR stars have been shown to present LPV patterns characteristic of what is expected from a wind with CIRs: WR 6 (WN 5; Morel et al. 1997), WR 134 (WN 6; Morel et al. 1999) and WR 1 (WN 4; Chené \& St-Louis 2010).

In a survey of spectroscopic variability of a magnitude-limited sample of WR stars, St-Louis et al. (2009, hereafter SLC) and Chené \& St-Louis (2011, hereafter CSL) found the WC 9 subclass to show particularly high levels of variability. In fact, all WC 9 stars in their sample except one, WR 103, showed variability levels larger than $\sim 5$ per cent of the line flux, while early-type single WC stars showed little to no variability at all ( $\leq 3$ per cent). Furthermore, the nature of

\footnotetext{
${ }^{1}$ Although some radial velocity variations have been found by Williams, van der Hucht \& Rauw (2005), a more complete radial velocity study is needed to characterize the orbital motion of the stars in WR 69.
}

the spectral variations appeared different from the ones generated by blobs, which are normally at a much lower level. The subpeaks superposed on the broad emission lines of WC 9 stars appeared to be much larger with respect to the total line width. Finally, some of the variability detected was on a relatively short time-scale (days), incompatible with a long-period binary.

The goal of this work is to characterize the variability of presumably single WC 9 stars and search for clues to identify the dominating mechanism responsible for the formation of dust in the wind of single WC 9 Stars. Among the questions on which we aim to shed more light on are: Are the short time-scale variations periodic? If so, are they related to CIRs or do they reveal the presence of a close companion? Indeed, to explain by which mechanism WC 9 stars make dust, Williams \& van der Hucht (1992) suggested that all WC 9 stars are binaries. Alternatively, if the variations are due to CIRs, why are they more present in this WR subtype compared to the others? Section 2 presents our observations while our results are described in Section 3. A discussion together with our conclusions follows in Section 4.

\section{OBSERVATIONS}

\subsection{Description}

The observations were obtained with the 0.6-m Helen Sawyer Hogg telescope at the El Leoncito Observatory (ELO) in Argentina and the 1.6-m telescope of the Observatoire du Mont Mégantic (OMM) in Québec, Canada. We observed every WC 9 star not demonstrated to be a binary listed in the VIIth catalogue of galactic WR stars (van der Hucht 2001) that has a magnitude of 13.25 or brighter. Because all the WC 9 stars in our sample have negative declinations, the majority of our spectra came from the ELO campaign. For each star, we obtained $\sim 1$ spectrum every 1 or 2 d over 1 month. Only WR 106, WR 119 and WR 121, the stars with the least negative declinations, were observable from the OMM. We obtained four to five additional spectra per night for eight nights over a period of $16 \mathrm{~d}$. The ELO campaign was carried out during 28 nights between 2009 May 16 and June 15 using a grating giving a resolution of $\Delta \lambda=2.91 \AA$ ( 3 pixels) and a spectral range of 4700-6100. The OMM campaign was from 2011 June 12 to June 28 for a total of eight clear nights of observations. We used a grating yielding a slightly higher resolution $\Delta \lambda=2.67 \AA$ ( 3 pixels) and a spectral range of 3800-6100 $\AA$. However, the sensitivity of the detector was too low shortward of $\sim 4700 \AA$ to provide a usable signal. For both observing runs, the slit width was roughly adjusted to the nightly seeing, which was typically $1.5-2$ arcsec in order to maximize throughput but maintain a stable spectral resolution. Details of our targets are given in Table 1, in which the stars' name, spectral type, number of spectra obtained and average $\mathrm{S} / \mathrm{N}$ for each run are listed. The $\mathrm{S} / \mathrm{N}$ was obtained from the standard deviation of a short continuum region around its median value.

\subsection{Reduction and extraction}

The spectra were reduced in the usual way using the $\mathrm{IRAF}^{2}$ software package. First a bias frame was subtracted, and then each image was

\footnotetext{
${ }^{2}$ IRAF is distributed by the National Optical Astronomy Observatories (NOAO), which is operated by the Association of Universities for Research in Astronomy, Inc. (AURA), under cooperative agreement with the National Science Foundation (NSF)
} 
Table 1. Our sample of WC 9 stars.

\begin{tabular}{|c|c|c|c|c|c|c|}
\hline \multirow[b]{2}{*}{ Name } & \multirow[b]{2}{*}{$\begin{array}{l}\text { Spectral } \\
\text { type }\end{array}$} & \multirow[b]{2}{*}{$\begin{array}{c}\text { Magnitude }{ }^{a} \\
\text { (v) }\end{array}$} & \multicolumn{2}{|c|}{ ELO run } & \multicolumn{2}{|c|}{ OMM run } \\
\hline & & & $\begin{array}{c}\text { Number of } \\
\text { spectra }\end{array}$ & $\begin{array}{c}\text { Average } \\
\text { S/N (continuum) }\end{array}$ & $\begin{array}{c}\text { Number of } \\
\text { spectra }\end{array}$ & $\begin{array}{c}\text { Average } \\
\text { S/N (continuum) }\end{array}$ \\
\hline WR 53 & WC $8 / 9 \mathrm{~d}^{b, c}$ & 10.88 & 22 & 25 & $\ldots$ & $\ldots$ \\
\hline WR 69 & $\mathrm{WC} 9 \mathrm{~d}^{b}$ & 9.43 & 22 & 43 & $\ldots$ & $\ldots$ \\
\hline WR 81 & WC $9^{b}$ & 12.71 & 20 & 15 & $\ldots$ & $\ldots$ \\
\hline WR 88 & $\mathrm{WC} 9^{b}$ & 13.25 & 19 & 12 & $\ldots$ & $\ldots$ \\
\hline WR 92 & $\mathrm{WC} 9^{b}$ & 10.43 & 22 & 27 & $\ldots$ & $\ldots$ \\
\hline WR 103 & $\mathrm{WC} 9 \mathrm{~d}^{a}$ & 8.86 & 20 & 36 & $\ldots$ & $\ldots$ \\
\hline WR 106 & $\mathrm{WC} 9 \mathrm{~d}^{a}$ & 12.33 & 16 & 19 & 18 & 65 \\
\hline WR 119 & $\mathrm{WC} 9 \mathrm{~d}^{b}$ & 12.41 & 17 & 17 & 26 & 69 \\
\hline WR 121 & WC $9 \mathrm{~d}^{a}$ & 12.41 & 16 & 15 & 24 & 69 \\
\hline
\end{tabular}

Notes. ${ }^{a}$ Spectral types and $v$ magnitudes are from van der Hucht (2001).

${ }^{b}$ Spectral types are from Williams \& van der Hucht (2000).

${ }^{c}$ Note that Crowther, De Marco \& Barlow (1998) have re-classified this star as WC 9 following their revision of the WC and WO classification criteria. Here, we retain the Williams \& van der Hucht (2000) spectral type (see their section 3.1).

divided by a flat-field. The wavelength calibration was carried out using an $\mathrm{He}-\mathrm{Ar}+\mathrm{Ne}$ calibration lamp for both runs. Special care was taken for the normalization of the spectra. First, for each star, a mean of all the spectra was computed. For WR 106, WR 119 and WR 121, a mean was made for each telescope separately. Then, each spectrum was divided by the mean and the ratio was fitted with a low-order Legendre polynomial (between fourth and eighth orders). Each original spectrum was then divided by its corresponding fit, bringing the continuum of each spectrum at the same level. For WR 106, WR 119 and WR 121, the spectra from each run were put at the same level by combining the run means and by repeating the procedure described above. The mean of all the spectra (or the mean of the run means for WR 106, WR 119 and WR 121) was then fitted with a first-order cubic spline in the few available continuum regions (4759-4769, 4885-4893, 4974-4991, 5394-5397, 55455576 and 595-6040 $\AA$ ) and each individual spectrum was divided by this fit to complete the continuum normalization.

\subsection{Wavelength calibration corrections}

For the OMM campaign, all our spectra were realigned using the $\mathrm{Na}$ I $\lambda 5890$ interstellar line. These narrow features were measured with sufficient precision so the standard deviation of the interstellar line positions on the realigned spectra is $\sim 0.03 \AA$. This value is adopted as the measurement error for those spectra. For the ELO campaign, we were only able to measure the position of the interstellar lines for the brightest stars: WR 69 and WR 103. For those stars, the standard deviations of the realigned interstellar lines are $\sim 0.07$ and $\sim 0.14 \AA$ for WR 69 and WR 103, respectively. The other stars have a typical wavelength calibration error of $\sim 0.45 \AA$. This value also corresponds to the standard deviation of the position of the sodium lines of WR 69 and WR 103 before the correction.

\section{VARIABILITY SEARCH}

\subsection{Temporal variance and $\sigma$ spectra}

To characterize the variability of our sample of WC 9 stars, the temporal variance spectrum (TVS) was calculated for each data set using the formalism of Fullerton, Gies \& Bolton (1996). More specifically, assuming that the noise in our sets of $N$ spectra is governed by a reduced $\chi^{2}$ distribution, we calculated the $\Sigma$ spectrum that indicates the level of significant variability at each wavelength using

$\Sigma_{j}(99$ per cent $)=\sqrt{\frac{(\mathrm{TVS})_{j}}{\sigma_{0}^{2} \chi_{N-1}^{2}(99 \text { per cent })}}$,

where $\sigma_{0}$ is a standardized dispersion based on the noise in the continuum. A spectrum that reaches a $\Sigma_{j}$ value of 2 , for example, is variable at the $2 \sigma$ level.

We found that it is more accurate to calculate the TVS using different continuum regions because the sensitivity of the CCD varies with wavelength and therefore the corresponding noise level in the continuum is not uniform. We calculated the TVS for 4700$5225 \AA$ using the continuum from $4760-4770 \AA$, the TVS for $5226-$ $5760 \AA$ using the continuum from 5510-5520 $\AA$ and the TVS for 5761-6000 ̊ using the continuum from 5930-5940 A.

Since the TVS compares the variation of the line flux to the noise level of the neighbouring continuum, it only provides limited physical information when studying spectral lines. A quantity that is more interesting for that purpose is the fraction of the line flux that is variable, the $\sigma$ spectrum. This quantity was introduced by SLC and is defined as

$\sigma_{j}($ per cent $)=\frac{\left(\mathrm{TVS}_{\mathrm{mod}}\right)_{j}^{1 / 2}}{\bar{S}_{j}-1}$,

where TVS $_{\text {mod }}$ is a modified TVS that compares the variance at pixel $j$ with the noise at that same pixel rather than to neighbouring continuum and $\bar{S}_{j}$ is the weighted mean continuum-normalized flux at wavelength $j$. This quantity is only defined in emission lines where the TVS is above the threshold level of $\sigma_{0}^{2} \chi_{N-1}^{2}$ (99 per cent). For fainter transitions, large parts of the line have a denominator too close to zero and the numerator is anyhow dominated by the noise. We therefore restrained our calculation of $\sigma$ to the emission lines having a relative intensity of at least 1.5 . For strong lines like $\mathrm{C}$ III $\lambda 5696$, we also restrained $\sigma$ to the full width at half-maximum (FWHM) of the line to avoid similar effects.

The $\Sigma$ (99 per cent) (bottom), $\sigma$ (middle) and mean (top) spectra are plotted for each star of our sample in Figs 1-6. We only show the entire spectral range for WR 69 (the second brightest star in our sample) because it is the only one observed during the ELO campaign that shows variability above the noise threshold for lines other than C III $\lambda$ 5696. WR 106, WR 119 and WR 121 also show variability for other lines, but only for the OMM spectra, which 


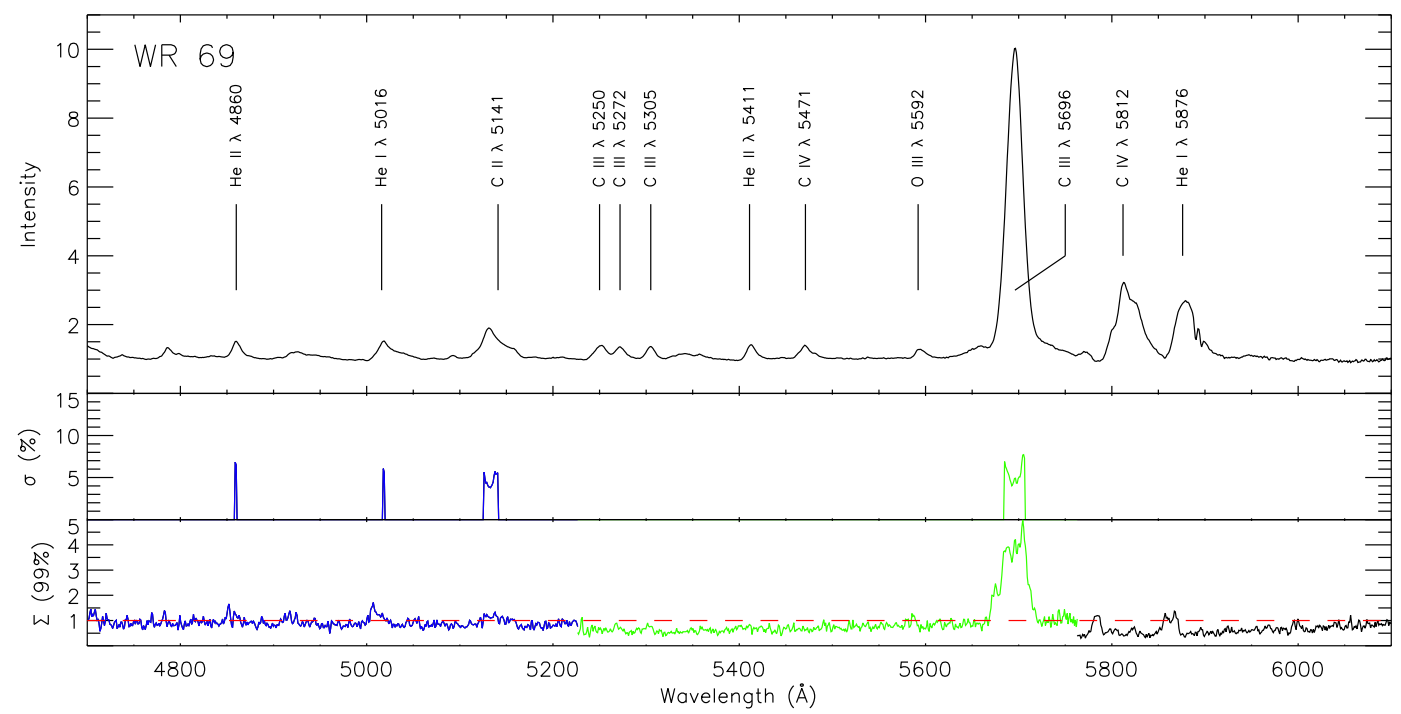

Figure 1. Top: mean spectrum of WR 69 for the entire ELO run. Middle: $\sigma$ spectrum. Bottom: $\Sigma(99$ per cent) spectrum. The horizontal dashed line is the variability threshold for $1 \sigma$ at a 99 per cent confidence level. The TVS is separated in three parts (hence the different colours) each using distinct continuum regions for reference (see the text).

have a higher $\mathrm{S} / \mathrm{N}$. The absence of significant variability outside the C III $\lambda 5696$ line for the ELO data (with the exception of WR 69) can most likely be explained by the modest $\mathrm{S} / \mathrm{N}$ of our spectra (see Table 1).

SLC and CSL observed a total of 64 apparently single WR stars with four to five spectra per target. One of their conclusions was that all observed lines for a given star show roughly the same level of variability. Note that they had, however, only two strong lines available for each star. We obtain similar results for WR 69 (see Fig. 1). We find that all major emission lines (He II $\lambda 4860$, He I $\lambda 5016, \mathrm{C}_{\text {II }}$ $\lambda 5130$ and $\left.C_{\text {III }} \lambda 5696\right)$ vary at a level of $\sigma \sim 5-6$ per cent. Furthermore, in general the lines listed above do vary synchronously. Variations are most easily seen in the $\mathrm{C}$ III $\lambda 5696$ emission profile as it is the strongest. More specifically, excesses on the red side of the He II $\lambda 4860, \mathrm{C}$ III $\lambda 5130$ and C III $\lambda 5696$ lines appear simultaneously while deficits of emission coincidently appear on the blue side of the $\mathrm{He} \mathrm{I} \lambda 5016$ and $\mathrm{C}$ III $\lambda 5696$ lines. This indicates that even if the lines are formed at different radii in the wind, they are equally affected by the physical mechanism generating the variability, hinting that the cause of the changes is a global wind effect. Another result that is found in both studies is that the values of $\Sigma$ ( 99 percent) are generally higher for the P Cygni absorption components than for their corresponding emission components. This can be clearly seen, for example, in the He II $\lambda 4860, \mathrm{He}_{\mathrm{I}} \lambda 5016, \mathrm{C}$ IV $\lambda 5812$ and He I $\lambda 5876$ lines of WR 69 (see Fig. 1). Since the absorption part of P Cygni profiles comes from a smaller volume of the wind, it suffers less from cancelling effects of large-scale changes in density or ionization arising in different parts of the wind.

Finally, we wanted to investigate the effect of using a limited number of spectra to assess the level of variability (in the case of SLC and CSL, only five). To do so, we have calculated the $\sigma$ spectrum with a series of 5,10 and 15 randomly chosen spectra for each star of our sample. This process was then repeated five times for each series. For each star, we present in Table 2 the mean values and standard deviations ${ }^{3}$ of $\sigma_{j}$ over the FWHM of the

\footnotetext{
${ }^{3}$ Note that these are the standard deviations of each series from the mean $\sigma$ value of the five repetitions. This is not to be confused with the standard
}

C III $\lambda 5696$ emission line, $\langle\sigma$ (per cent $)\rangle$, for each series as well as the value obtained with all the spectra, highlighted in bold. Note that this process was not as useful for the OMM data because we generally obtained three to five consecutive spectra each night over a total of eight nights. Thus, as we will describe later, because we find little variability within a given night, the OMM observations yielded fewer significantly different spectra than for the ELO run, even though we have more of them. A closer inspection of Table 2 confirms this, as it reveals that the $\sigma$ values for the OMM stars do not vary very much as a function of the number of spectra used. When taking the results for all stars into consideration, the conclusion that can be drawn concerning the $\sigma$ value with respect to the number of spectra is that using five spectra instead of $\sim 20$ leads to at most a difference in the estimated variability level of $\sim 1$ per cent.

To illustrate this, we plot in Fig. 7 the $\sigma$ spectrum for the C III $\lambda 5696$ of WR 69 for each series of randomly chosen spectra. Although the shape of the profile presents more differences from one series of five spectra to the other, the average value of $\sigma$ over the line is not very affected. Note that for the series of 15 spectra, the data sets are becoming less and less independent from each other. Based on previous experiences with WR 6 and WR 134, two stars for which CIRs have been detected, SLC assumed that four to five spectra well sampled in time would be enough to identify new CIR candidates. Based on the results presented above, we conclude that this is a relatively good assumption but that the detailed shape of the $\sigma$ profile for such a small number of spectra should not be overinterpreted. Note, however, that other effects can affect the level of variability derived from a series of spectra (see Section 3.3).

From Figs 1 to 7, we note that for many WC 9 stars, the $\sigma$ profile for the C III $\lambda 5696$ line for a given star indicates a higher level of relative variability in the wings than in the core of the line. Such a profile can be generated if the line profiles are misaligned when comparing them to one another. However, in this case we believe that this is not a valid explanation as if it were so, the effect would be greatly reduced for the profiles we were able to align correctly using

deviation of each $\sigma$ value obtained by averaging the pixel values within the FWHM of the line. 

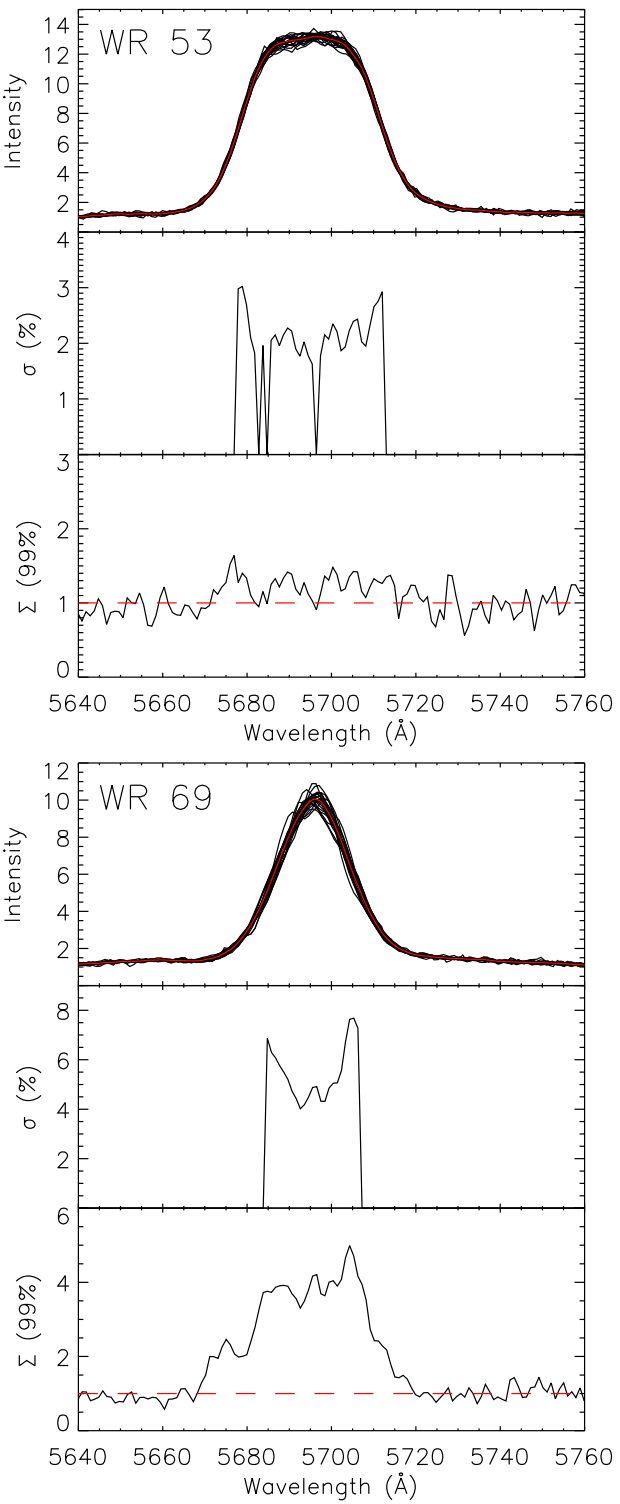

Figure 2. (a) Top: a superposition of the spectra of WR 53 for the entire ELO run, centred on the $\mathrm{C}$ III $\lambda 5696$ line with the mean spectrum overplotted in red. Middle: $\sigma$ spectrum. Bottom: $\Sigma$ (99 per cent) spectrum. (b) Same as (a) for WR 69 .

narrow interstellar lines, i.e. WR 69, WR 103 and the ones obtained at the OMM. This is not what is found (see Fig. 5 for example). Another explanation, which is more plausible, is that the particular $\sigma$ profile shape finds its origin in the nature of the variability itself. Inspection of series of line profiles leads us to believe that it is caused by what can be qualified as 'breathing' of the spectral line, i.e. a slight change in its total width with a smaller level of variability in its peak intensity. This can be caused, for example, by a small change in the terminal velocity of the wind or in the extent of the line-formation zone. Such changes would produce a very high level of relative variability in the very steepest parts of the line, its wings. This can be seen, for example, by comparing the superposed profiles of WR 53 and WR 69 in Fig. 2. The width of the superposition of all spectra is much higher in the wings of the line for WR 69 than for WR 53, indicating a higher level of variability in the $\sigma$ profile wings for that star.
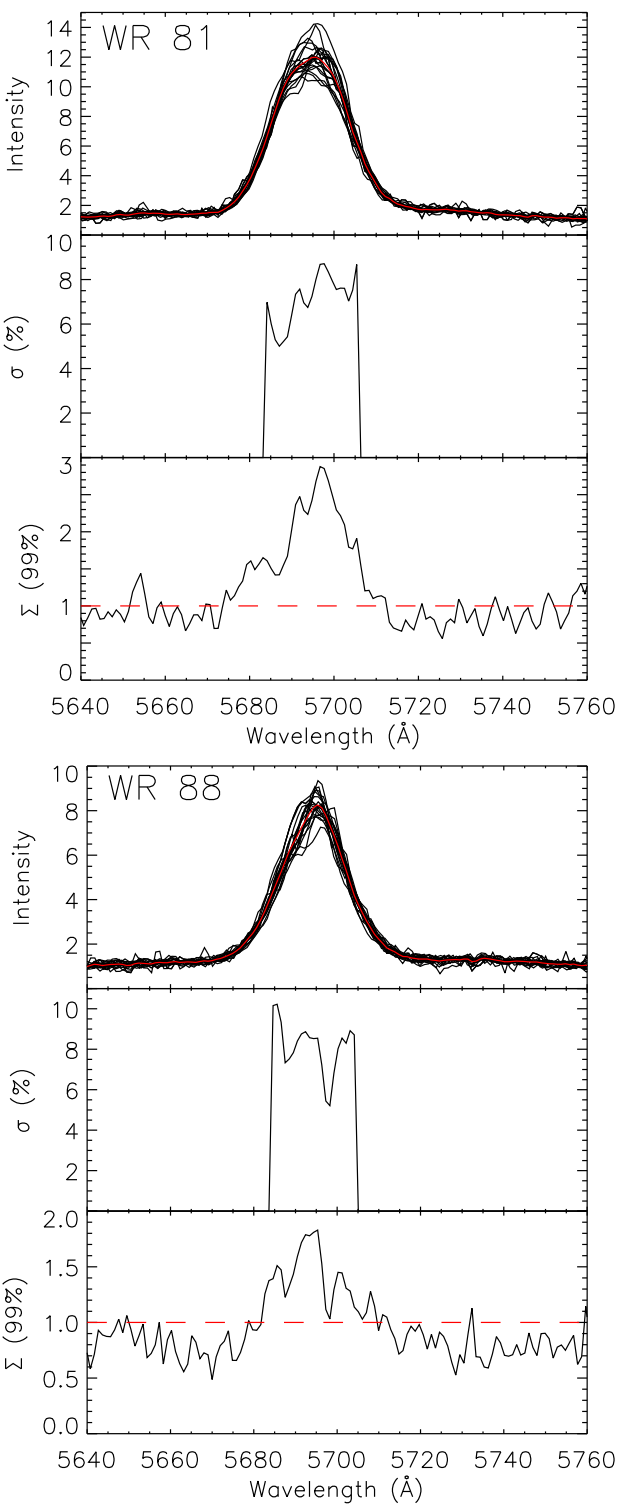

Figure 3. Same as Fig. 2(a) for (a) WR 81 and (b) WR 88.

\subsection{Wavelet analysis}

Another way to characterize the variability of our sample of WC 9 stars is by analysing the size distribution of the structures in the residuals obtained by subtracting the mean profile from each individual spectrum. We used the wavelet transform to quantify the various components and to obtain the mean line-of-sight velocity dispersion of line profile variability subpeaks.

Following Lépine \& Moffat (1999, hereafter LM), we define the residual spectra centred on the $\mathrm{C}$ III $\lambda 5696$ line as

$R\left(\xi, t_{i}\right)=S\left(\xi, t_{i}\right)-\frac{1}{N} \sum_{i=1}^{N} S\left(\xi, t_{i}\right)$,

where $S\left(\xi, t_{i}\right)$ is the spectral time series, $\xi=\left(\lambda-\lambda_{0}\right) \lambda_{0}^{-1} c$ with $\lambda_{0}=5696 \AA$ is the line-of-sight velocity and $N$ is the number of spectra. The continuous wavelet transform ${ }^{4}$ of each residual spectrum, denoted $\widetilde{R}\left(\xi, \sigma_{\xi}, t_{i}\right)$, is then calculated individually and averaged

\footnotetext{
${ }^{4}$ See appendix A of LM for a detailed explanation of wavelet analysis.
} 

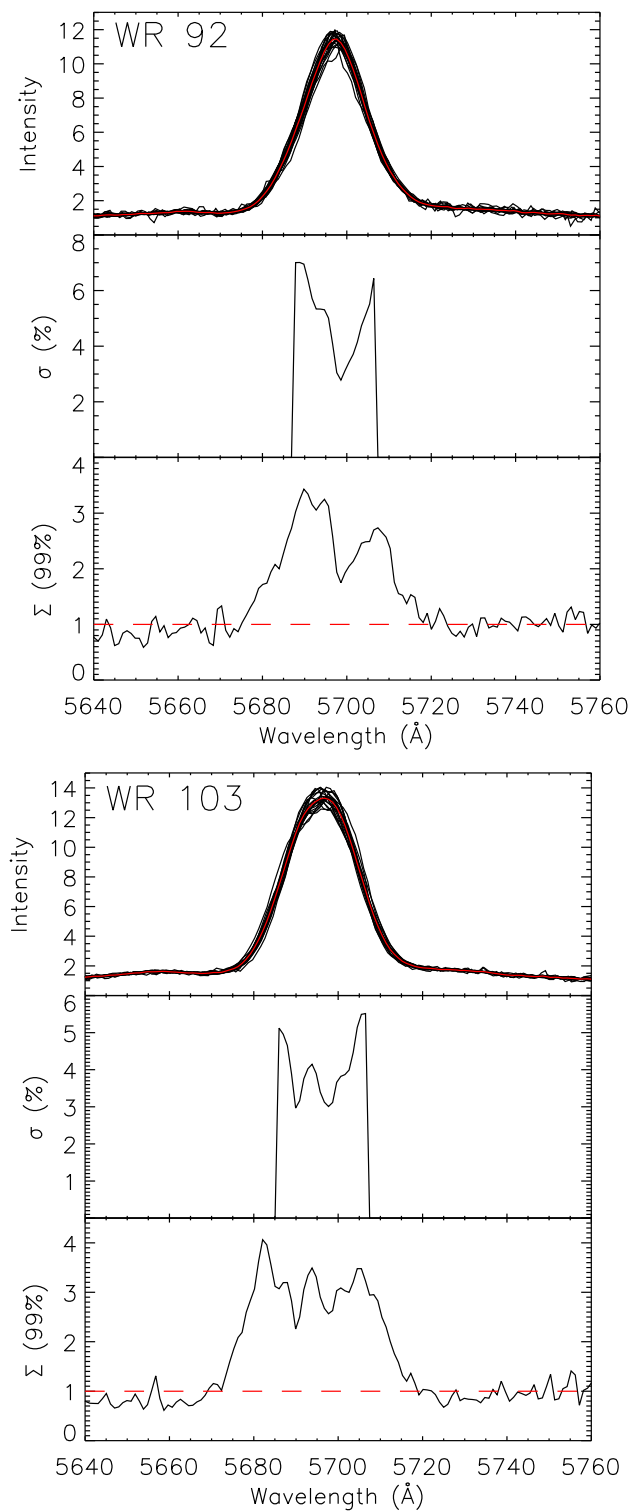

Figure 4. Same as Fig. 2 (a) for (a) WR 92 and (b) WR 103.

over the entire data set to yield $\left\langle\widetilde{R}\left(\xi, \sigma_{\xi}\right)\right\rangle$, the mean wavelet transform given by

$\left\langle\widetilde{R}\left(\xi, \sigma_{\xi}\right)\right\rangle^{2}=\frac{1}{N} \sum_{i=1}^{N}\left[\widetilde{R}\left(\xi, \sigma_{\xi}, t_{i}\right)\right]^{2}$.

A plot of $\left\langle\widetilde{R}\left(\xi, \sigma_{\xi}\right)\right\rangle^{2}$ for each star is available as online material accompanying this paper.

The mean wavelet transform characterizes to what extent features of a given width $\sigma_{\xi}$ are present in the residuals as a function of $\xi$, their line-of-sight velocity. In order to identify the dominant scale, i.e. the one that produces the maximum power for the entire emission line, we obtained the wavelet power spectrum (WPS), $\left\langle\widetilde{R}\left(\sigma_{\xi}\right)\right\rangle_{\mathrm{LPV}},{ }^{5}$ by integrating the mean wavelet power spectrum over the location of the features in velocity space, $\xi$, within the FWHM of the line to avoid edge effects. The position of the maximum of $\left\langle\widetilde{R}\left(\sigma_{\xi}\right)\right\rangle_{\mathrm{LPV}}$,

${ }^{5}$ Here, the LPV subscript stands for line profile variations.
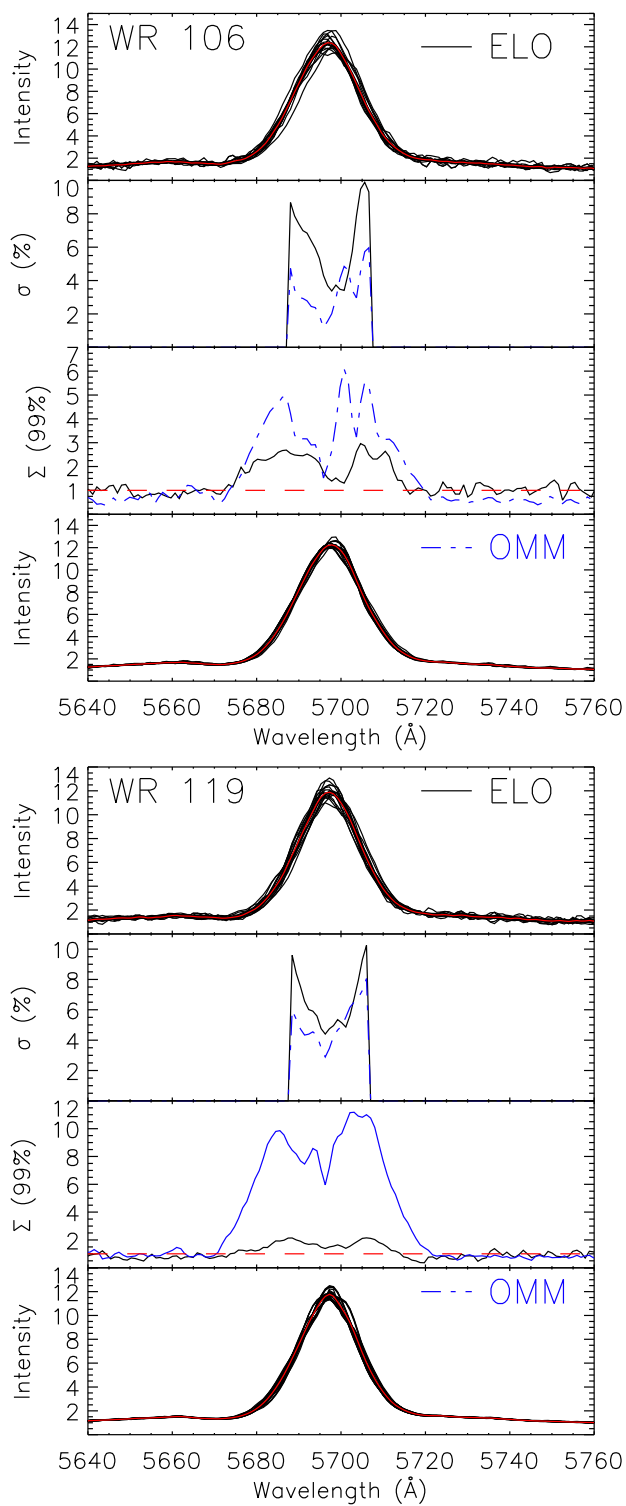

Figure 5. (a) Top: a superposition of the spectra of WR 106 for the entire ELO run, centred on the $\mathrm{C}$ III $\lambda 5696$ line with the mean spectrum overplotted in red. Second from top: $\sigma$ spectrum for the ELO run (black solid line) and for the OMM run (blue dot-dashed line). Second from bottom: $\Sigma$ (99 per cent) spectrum for the ELO run (solid line) and for the OMM run (blue dot-dashed line). Bottom: same as top panel but for the OMM run. (b) Same as (a) for WR 119.

denoted $\overline{\sigma_{\xi}}$, yields a measure of the mean line-of-sight velocity dispersion of the subpeaks.

Note that a large-scale component at $\sim 1000 \mathrm{~km} \mathrm{~s}^{-1}$ was introduced in the wavelet spectrum by the mean subtraction process described above. This component corresponds to the scale of the Mexican-hat wavelet ${ }^{6}$ that fits the length of our residual spectra centred on the $\mathrm{C}$ III $\lambda 5696$ line. This windowing effect appears in the wavelet spectrum because the subtraction of the mean profile from the spectra introduces a dip (or bump) as wide as the line due to a slight difference between the intensity of the spectra and that of the mean profile. We were able to isolate this artefact by carrying out a boxcar smoothing of the residuals to model all features that

${ }^{6}$ The second derivative of a Gaussian function. 


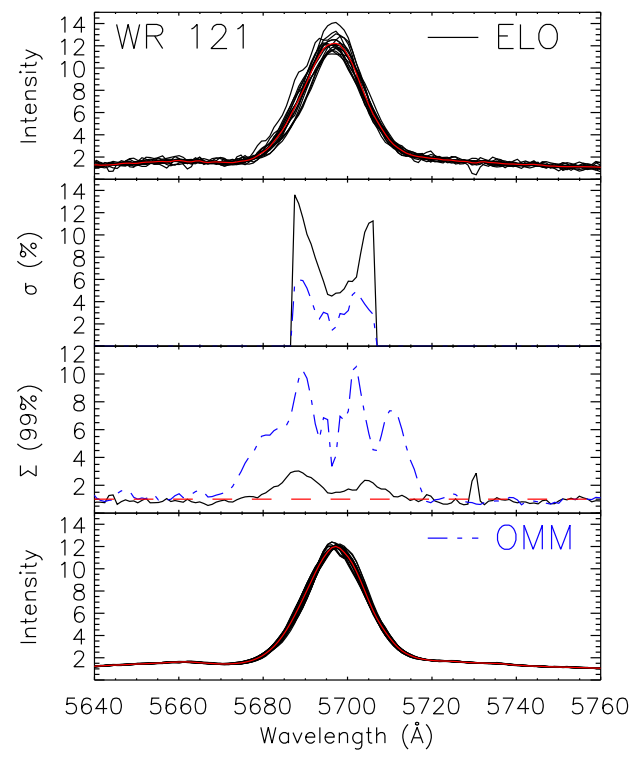

Figure 6. Same as Fig. 5(a) for WR 121.

have a characteristic scale larger than or equal to $\sim 1000 \mathrm{~km} \mathrm{~s}^{-1}$. We then subtracted the smoothed function from the residuals before calculating the wavelet transform.

We present in Fig. 8 the WPS, $\left\langle\widetilde{R}\left(\sigma_{\xi}\right)\right\rangle_{\mathrm{LPV}}$, for the spectral time series of each star in our sample for the $C_{\text {III }} \lambda 5696$ line. Even though the analysis from both the ELO and OMM observing runs yielded much more similar results than for the TVS, we processed each data set separately for WR 106, WR 119 and WR 121 for completeness. The only star that is common between LM's sample and ours is WR 103. If we compare our wavelet power spectrum $\left\langle\widetilde{R}\left(\sigma_{\xi}\right)\right\rangle_{\mathrm{LPV}}$ with theirs for that star (see bottom right-hand panel of Fig. 8 and their fig. 4), we find very good agreement, except for the fact that both studies do not cover the same range of $\sigma_{\xi}$ due to the very different spectral resolutions. Because the spectral resolution of our data is lower, we cannot resolve features that have a characteristic scale smaller than $\sigma_{\xi} \sim 100 \mathrm{~km} \mathrm{~s}^{-1}$ whereas LM's data with a dispersion of $0.1 \AA$ pixel $^{-1}$ enable them to resolve structures down to a scale of $\sim 6 \mathrm{~km} \mathrm{~s}^{-1}$. Both studies find a maximum around $\sigma_{\xi} \sim$ $10^{2.1-2.2}$ with a kink around $\sigma_{\xi} \sim 10^{2.7}$ and a broad shoulder towards larger structures. Actually, all the stars of LM's sample, except WR 134, show a maximum at a similar mean velocity dispersion of $\overline{\sigma_{\xi}} \sim 100-125 \mathrm{~km} \mathrm{~s}^{-1}$. Our data for these WC 9 stars show a typical value of $\overline{\sigma_{\xi}}$ that is slightly higher at around $175-200 \mathrm{~km} \mathrm{~s}^{-1}$.

Note that LM's observations consisted of roughly one spectrum every hour over an interval of $\sim 8 \mathrm{~h}$ for three or four consecutive nights while our ELO data consist of one spectrum every 1 or sometimes $2 \mathrm{~d}$ over a 1-month period. While LM's spectral and temporal resolutions make their time series ideal to study LPVs on short time-scales, it is possible that variations on a time-scale of days to weeks are missed. If there is a rather large-scale subpeak that moves slowly across an emission line in a series of spectra obtained very closely spaced in time, the subpeak would roughly be at the same place and visible in all of them. Therefore, the structure will not be averaged out of the mean spectrum, which means that it will not appear in the residuals. On the other hand, if spectra are taken

Table 2. $\sigma$ Spectrum of the $\mathrm{C}_{\text {III }} \lambda 5696$ line for our sample of WC 9 stars.

\begin{tabular}{|c|c|c|c|c|c|c|c|}
\hline Star & $\begin{array}{c}\text { Number of } \\
\text { spectra }\end{array}$ & $\begin{array}{l}\text { Average } \\
\text { value }\end{array}$ & $\begin{array}{l}\text { Standard } \\
\text { deviation }\end{array}$ & Star & $\begin{array}{c}\text { Number of } \\
\text { spectra }\end{array}$ & $\begin{array}{c}\text { Average } \\
\text { value }\end{array}$ & $\begin{array}{l}\text { Standard } \\
\text { deviation }\end{array}$ \\
\hline \multirow[t]{5}{*}{ WR 53} & 5 & 2.7 & 0.2 & & 5 & 6.0 & 3.0 \\
\hline & 10 & 2.5 & 0.2 & WR 106 & 10 & 6.2 & 2.0 \\
\hline & 15 & 2.4 & 0.1 & (ELO) & 15 & 7.1 & 0.1 \\
\hline & All (22) & 2.2 & $\ldots$ & & All (16) & 6.4 & $\ldots$ \\
\hline & M2014 & $<1.5(5 \mathrm{~d})$ & $\ldots$ & & M2014 & $6.5(4 \mathrm{yr})$ & $\ldots$ \\
\hline \multirow[t]{5}{*}{ WR 69} & 5 & 4.5 & 0.5 & & 5 & 3.1 & 0.4 \\
\hline & 10 & 5.3 & 0.8 & WR 106 & 10 & 3.3 & 0.1 \\
\hline & 15 & 5.6 & 0.3 & (OMM) & 15 & 3.3 & 0.1 \\
\hline & All (22) & 5.5 & $\ldots$ & & All (18) & 3.4 & $\ldots$ \\
\hline & & & $\ldots$ & & M2014 & 6.5 & $\ldots$ \\
\hline \multirow[t]{5}{*}{ WR 81} & 5 & 6.3 & 1.4 & & 5 & 6.2 & 0.4 \\
\hline & 10 & 6.4 & 0.9 & WR 119 & 10 & 6.4 & 0.7 \\
\hline & 15 & 6.7 & 0.5 & (ELO) & 15 & 6.2 & 0.4 \\
\hline & All (20) & 7.1 & $\ldots$ & & All (17) & 6.5 & $\ldots$ \\
\hline & M2014 & $5.5(8 d)$ & $\ldots$ & & M2014 & $3.0(35 \mathrm{~d})$ & $\ldots$ \\
\hline \multirow[t]{5}{*}{ WR 88} & 5 & 9.3 & 1.1 & & 5 & 4.5 & 1.0 \\
\hline & 10 & 8.0 & 0.8 & WR 119 & 10 & 4.6 & 0.7 \\
\hline & 15 & 8.3 & 0.7 & (OMM) & 15 & 4.7 & 0.3 \\
\hline & All (19) & 8.2 & $\ldots$ & & All (26) & 5.1 & $\ldots$ \\
\hline & M2014 & $6.5(6 \mathrm{~d})$ & $\ldots$ & & M2014 & $3.0(35 \mathrm{~d})$ & $\ldots$ \\
\hline \multirow[t]{5}{*}{ WR 92} & 5 & 5.0 & 1.0 & & 5 & 7.7 & 1.1 \\
\hline & 10 & 4.8 & 0.4 & WR 121 & 10 & 8.0 & 0.7 \\
\hline & 15 & 5.4 & 0.2 & (ELO) & 15 & 7.9 & 0.2 \\
\hline & All (22) & 5.0 & $\ldots$ & & All (16) & 7.9 & $\ldots$ \\
\hline & M2014 & $5.1(6 d)$ & $\ldots$ & & M2014 & $5.0(35 \mathrm{~d})$ & $\ldots$ \\
\hline \multirow[t]{5}{*}{ WR 103} & 5 & 4.1 & 0.5 & & 5 & 3.9 & 0.7 \\
\hline & 10 & 4.3 & 0.2 & WR 121 & 10 & 3.6 & 0.3 \\
\hline & 15 & 4.3 & 0.1 & (OMM) & 15 & 3.7 & 0.10 \\
\hline & All (20) & 4.1 & $\ldots$ & & All (24) & 3.6 & $\ldots$ \\
\hline & M2014 & $4.0(7 \mathrm{~d})$ & $\ldots$ & & M2014 & $5.0(35 \mathrm{~d})$ & $\ldots$ \\
\hline
\end{tabular}



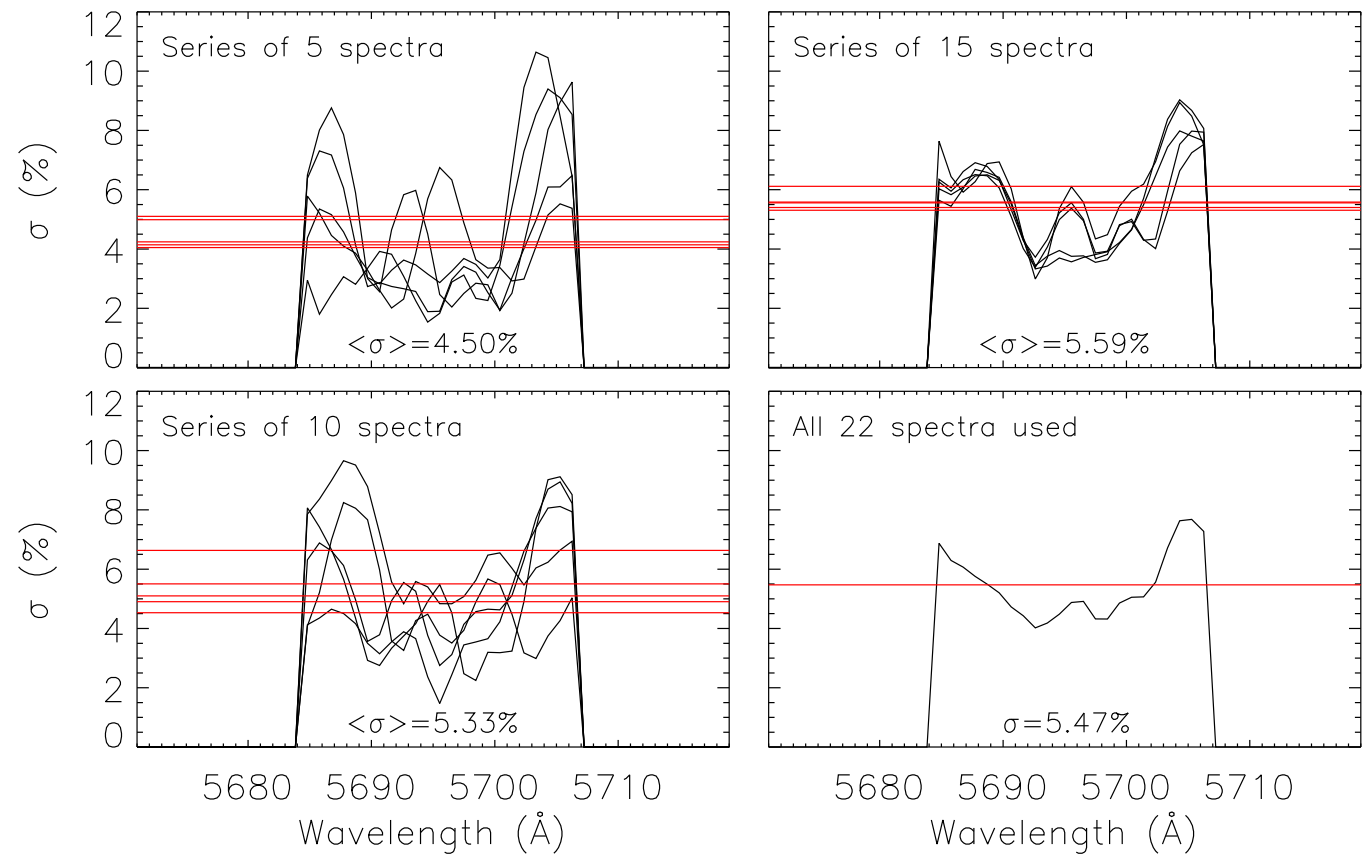

Figure 7. Top left: $\langle\sigma$ (per cent) $)\rangle$ spectrum for all the five-spectra series. The horizontal red lines indicate the corresponding $\sigma$ values. The mean value of $\sigma$ for the five-spectra series is indicated inside the plot. Bottom left: same plot but for the 10-spectra series. Top right: same plot but for the 15-spectra series. Bottom right: $\sigma$ spectrum calculated with all the available spectra.
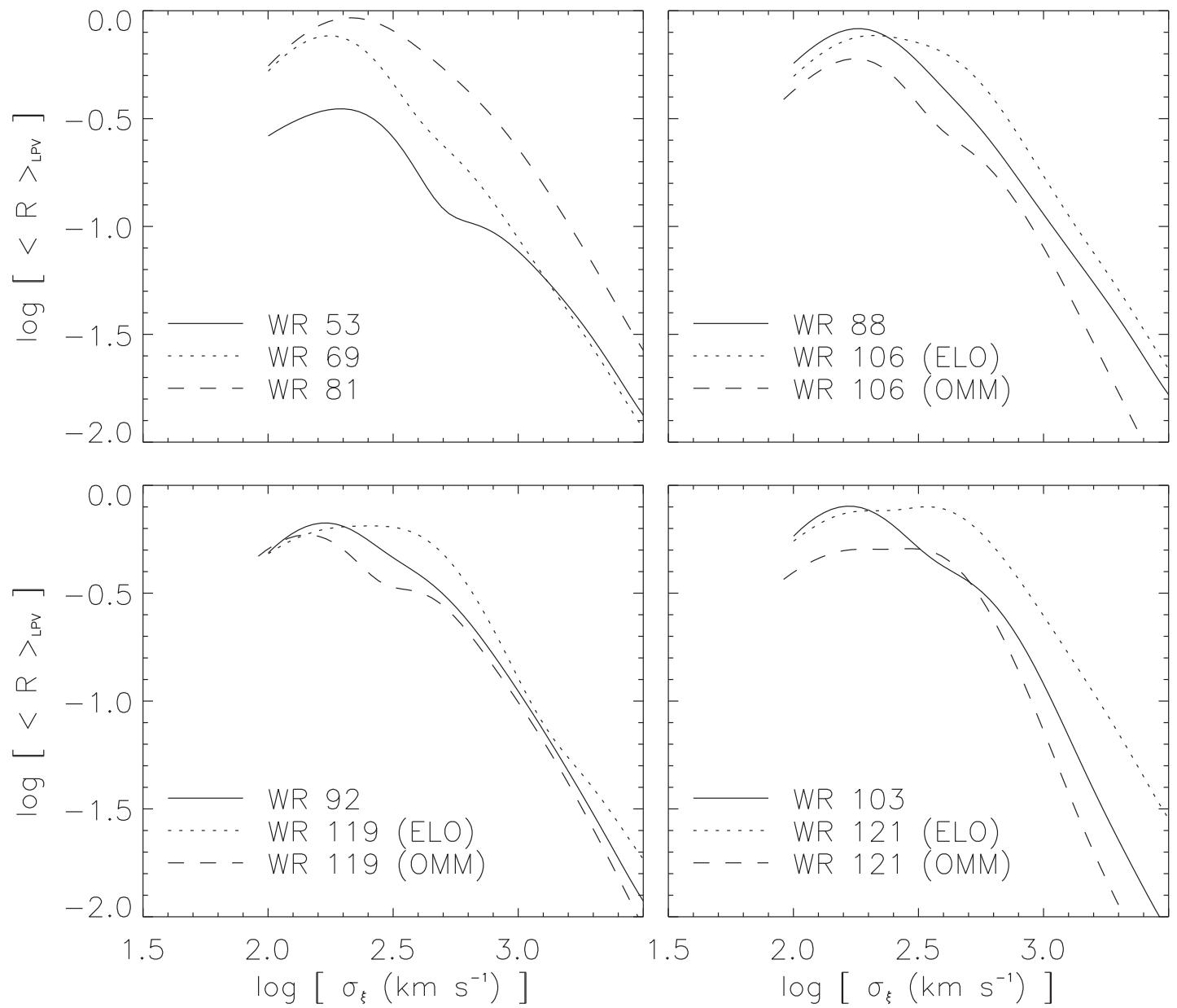

Figure 8. The WPS, $\left\langle\widetilde{R}\left(\sigma_{\xi}\right)\right\rangle_{\mathrm{LPV}}$, for the spectral time series in our data set. 
over a longer period of time, the mean spectrum would not contain much trace of the large-scale subpeak and therefore the residuals would. With a short 2.25-d period, it is not surprising that WR 134's large-scale pattern was able to be revealed in LM's observations.

\subsection{Time-scale and amplitude of the variability for individual stars}

For WR 106, WR 119 and WR121, we calculated $\Sigma$ (99 per cent) and $\sigma$ separately for both observing runs as it yielded slightly different results (Figs 5 and 6). It is clearly easier to detect the variability with the higher S/N OMM data and this is especially true for WR 119 and WR 121 where $\Sigma(99$ per cent) reaches 8-10 for the OMM spectra but barely 2-3 for the ELO spectra (see Fig. 5b). As expected, the $\sigma$ spectrum is however approximately the same (WR 119) or even higher (WR 106 and WR 121) for the ELO than for the OMM spectra. One likely explanation for the higher variability level of the ELO data is the different time sampling and total time coverage of each data set. The ELO data consist of roughly one spectrum every 1 or $2 \mathrm{~d}$ over a month, which covers a broader time span than the OMM data with three to six spectra per night for only $8 \mathrm{~d}$ over a $14-d$ period. If the variations were on a short time-scale $(\sim 2-14 \mathrm{~d})$, the $\sigma$ values should be the same for both observing runs since both would have a sufficient time window to detect them. However, if the variations take place over a greater time period, the $\sigma$ value obtained for the OMM run could easily be lower as they would cover only part of the variations. Also, if the variations were on an even shorter time-scale ( $\lesssim 1 \mathrm{~d}$ ), we would more likely observe a higher $\sigma$ value for the OMM spectra than for the ELO data for which we have only one spectrum per day. We therefore conclude that the variations for WR 106 and WR 121 occur on a time-scale of $>14 \mathrm{~d}$.

The $\langle\sigma$ (per cent $)\rangle$ values for each star of our sample can be found in Table 2 highlighted in bold. For comparison, we also include in this table the values of SLC and CSL compiled by Michaux et al. (2014, hereafter M2014), except for WR 69 which they did not observe. In general, the values tabulated by M2014 are smaller or very similar to those determined in this work. Various cases can be distinguished. For WR 53, WR 81 and WR 88, the data of CSL were obtained on a shorter time span (5-8 d) and their $\sigma$ values are also smaller, suggesting that the full variability of these stars occurs on time-scales of a few weeks rather than days. On the other hand, for WR 92 and WR 103, a time span of 6-7 d seems to have been sufficient to detect the full level of the line variability. In those cases, we conclude that the variability occurs on a time-scale of days. For WR 119 and WR 121, the five spectra of SLC seem to have been obtained too spread out in time ( $35 \mathrm{~d})$ to detect the full variability level that we observe whereas this seems not to have been a problem for WR 106. We conclude from these comparisons that the accuracy of the value of $\sigma$ describing the level of emission line variability depends on the time span over which the data have been obtained compared to the time-scale of the variability. As the latter cannot be known in advance, we caution that unless the star has been observed intensely over a long period of time, the $\langle\sigma$ (per cent) $\rangle$ value should be considered as lower limits. Even the values presented here could turn out to be lower limits as observations on a longer time span could potentially reveal a larger level of variability. In summary, with the data in hand, we find that the minimum time-scale over which the full extent of the variability level takes place is a few days (WR 92, WR 103) to 1-2 weeks (WR 119) to $\gtrsim$ few weeks (WR 53, WR 81, WR 88, WR 106, WR 121). For WR 69, we have no previous data set to compare to.
Note that some changes can occur on a much shorter time-scale than the values stated above. Actually, we will see in Section 3.4 that the spectrum can be extremely different from night-to-night and even on time-scale of hours. However, for the purpose of estimating the full extent of the variability levels, longer observing periods must be used.

In order to identify viable CIR candidates, which should show large-scale line variability, SLC adopted a threshold of $\sigma=5$ per cent to distinguish stars that display small-scale variability (SSV; $\lesssim 5$ per cent) from those that display large-scale variability (LSV; $\sigma \gtrsim 5$ per cent). Stars in the SSV category typically have a variability level of $\sigma \sim 2-3$ per cent. The origin of this variability is most likely wind inhomogeneities (e.g. Moffat et al. 1988; Lepine $\&$ Moffat 1999). Among the stars that fall into the LSV category are all the WC 9 stars, except WR 103, which only shows variability at a level of $\sim 4$ per cent and is therefore formally in the SSV category. All earlier type WC stars in their sample that show significant variability are also in the SSV category. We obtain results that are consistent with these conclusions. In particular, the only star in our sample that is not of WC 9 subtype, WR 53 (WC 8d), has a low $\langle\sigma($ per cent $)\rangle$ value of 2.20 per cent. We confirm that all WC 9 stars in the SLC and CSL sample (except WR 103 with $\sigma \approx 4$ per cent) have $\sigma$ values between 5.5 and 8.2 per cent. Note that this result on the spectral variability level of WR 53 is compatible with the conclusion of Williams \& van der Hucht (2000) that the spectrum of this star is sufficiently different from that of other WC 9 stars for them to adopt a WC8/9 spectral type.

In addition to the $\langle\sigma$ (percent) $\rangle$ values, CSL also presented a wavelet analysis of the significantly variable stars of their sample. They obtain $\overline{\sigma_{\xi}} \sim 100-200 \mathrm{~km} \mathrm{~s}^{-1}$ for the SSV stars and $\overline{\sigma_{\xi}} \sim$ $300-500 \mathrm{~km} \mathrm{~s}^{-1}$ for the non-WC 9 LSV stars. Their sample of WC 9 stars (all classified as LSV except WR 103) yielded intermediate $\overline{\sigma_{\xi}}$ values, around $\sim 150-300 \mathrm{~km} \mathrm{~s}^{-1}$. In Fig. 9, we present an updated version of CSL's fig. 23 , where the $\overline{\sigma_{\xi}}$ values are plotted as a function of $\langle\sigma$ (per cent $)\rangle$, the mean value of their $\sigma$ spectrum over the FWHM of available lines, which were compiled by M2014. We added to this figure the results of this study of WC 9 stars as red diamond symbols. We excluded the OMM data from the plot since the $\langle\sigma$ (per cent $)\rangle$ values are probably not as accurate as those of the ELO run due to a shorter time span.

As CSL, for WC 9 stars we find values for $\overline{\sigma_{\xi}}$ in the 150 $300 \mathrm{~km} \mathrm{~s}^{-1}$ range. Within the error bars all our values agree with theirs. On the other hand, our values of $\langle\sigma$ (per cent) $\rangle$ are either equal to or larger than the ones determined by CSL and SLC. This is most likely caused by the generally shorter time span over which the data of CSL and SLC were obtained combined with the actual time-scale of the variability. Nevertheless, the new data points we have added to this figure are still in agreement with the results of CSL who concluded that a higher value $\langle\sigma$ (per cent) $\rangle$ is generally associated with a higher $\overline{\sigma_{\xi}}$ value, as shown in Fig. 9, which suggest a possible link between these two quantities. This confirms CSL's statement that the variability could perhaps eventually be characterized by only one of these two quantities.

The case of WR 53 (WC 8d) is distinct from that of the WC 9 stars in our sample. This star shows a lower level of variability with $\sigma=2.20$ per cent. This, associated with the quasi-absence of variability in the line wings, suggests a different nature of the variability. Interestingly, the structures in its residuals show a mean velocity dispersion of $\overline{\sigma_{\xi}} \approx 200 \mathrm{~km} \mathrm{~s}^{-1}$, which is roughly what we obtain for other WC 9 stars but much larger than what LM obtained for small amplitude changes that are generally associated with clumps in the wind and that produce a 


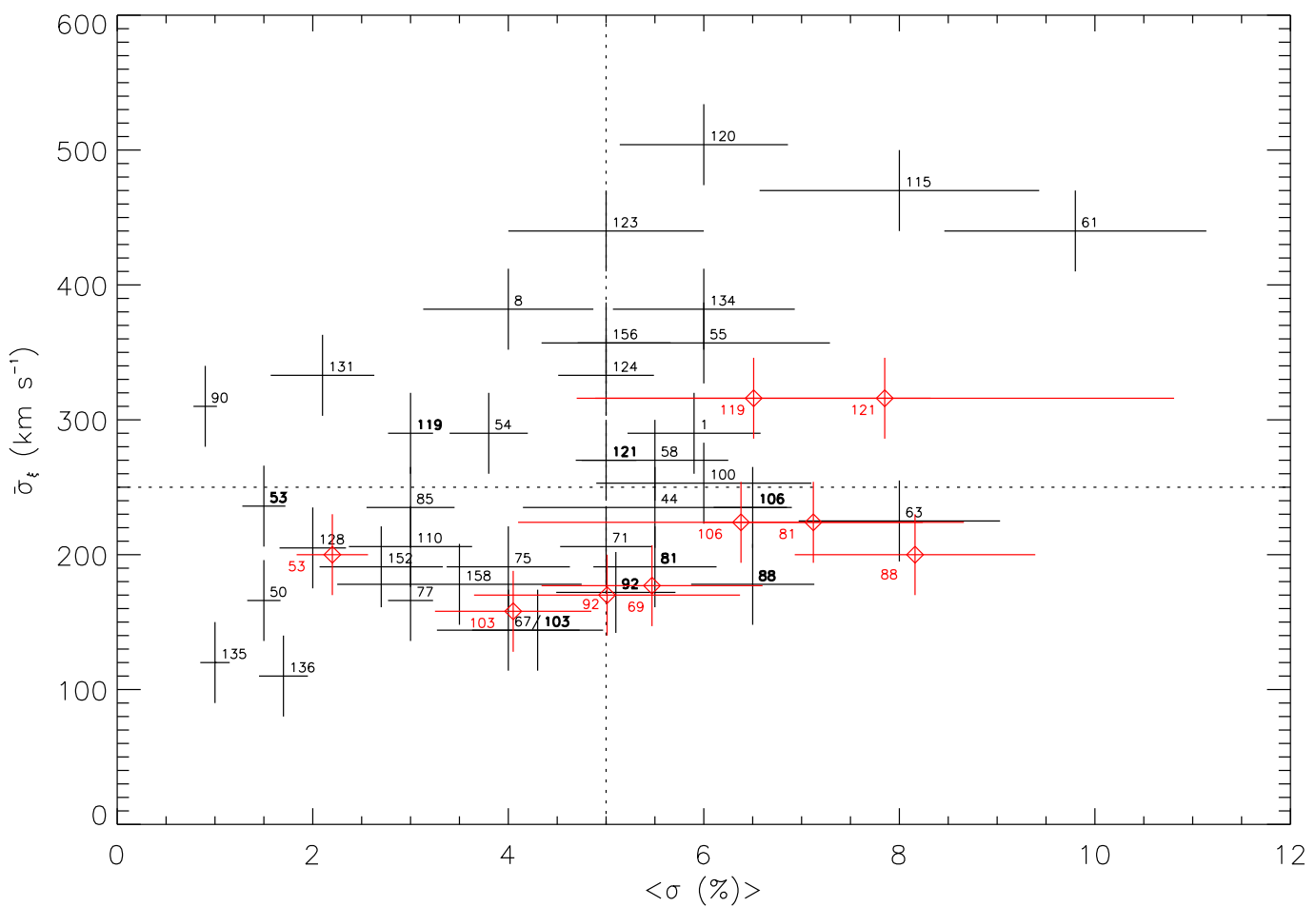

Figure 9. Maximum of the mean WPS, $\overline{\sigma_{\xi}}$ of all the significantly variable stars of CSL (plotted in black) and our sample of WC 9 stars (plotted in red diamonds) as a function of $\langle\sigma$ (per cent) $)$, the mean value of their $\sigma$ spectrum. For each star, marked in the plot by its WR number, a $1 \sigma$ error bar is plotted along both axes. For better comparison, we highlighted in bold the WC 9 stars of CSL. The vertical dotted line marks the limit of $\sigma=5$ per cent that separates SSV from LSV stars, as adopted by SLC. The horizontal one marks $\overline{\sigma_{\xi}}=250 \mathrm{~km} \mathrm{~s}^{-1}$.

small level of spectral variability such as the one we find here for this star.

\subsection{Quantitative line-profile analysis}

The $\sigma$ spectrum and the mean WPS that we calculated for the $\mathrm{C}$ III $\lambda 5696$ line of the stars of our sample enable us to characterize their variability in a qualitative manner. In order to get a more quantitative view of the variability and to search for signs of periodicity that could be related to large-scale wind structures like CIRs or to a hidden companion, we have computed the following integrated line quantities: equivalent widths (EW), radial velocities (RV), kurtosis and skewness of the $\mathrm{C}$ III $\lambda 5696$ line for every spectrum of all stars in our sample. We present the mean values and the standard deviations of these quantities for each star in Table 3 and the complete list in the on-line material (Table 4). We searched for periodicities in these measured quantities using two different methods: phasedispersion minimization (PDM) and by calculating a Lomb-Scargle periodogram.

We considered the limits of the line to be the points where we could trace a tangent oriented at $45^{\circ}$ from the horizontal. Of course, this gave slightly different limits for each star but it roughly corresponds to the wavelength interval $\Delta \lambda \sim 5675-5718 \AA$ for WC 9 stars. The stars in our sample display different levels of variability for the EW of the $\mathrm{C}$ III $\lambda 5696$ line but no clear periodicity was found. However, there is a clear correlation between the relative variation in the EW (sigma/mean) that range between 1.4 and 7.4 per cent and the average $\sigma$ (per cent) value across the line

Table 3. Average moments and standard deviations.

\begin{tabular}{|c|c|c|c|c|c|c|c|c|}
\hline \multirow{2}{*}{$\begin{array}{l}\text { Star } \\
\text { WR } 53\end{array}$} & \multicolumn{2}{|c|}{$\begin{array}{c}\text { Equivalent widths } \\
(\AA)\end{array}$} & \multicolumn{2}{|c|}{ Skewness } & \multicolumn{2}{|c|}{ Kurtosis } & \multicolumn{2}{|c|}{$\begin{array}{l}\text { Radial velocity } \\
\qquad\left(\mathrm{km} \mathrm{s}^{-1}\right)\end{array}$} \\
\hline & -424.3 & $(6.8)$ & 0.024 & $(0.010)$ & 2.306 & $(0.010)$ & 0.01 & $(8.62)$ \\
\hline WR 69 & -195.1 & $(4.9)$ & 0.006 & $(0.032)$ & 2.693 & $(0.035)$ & -0.03 & $(15.20)$ \\
\hline WR 81 & -244.7 & (15.8) & 0.024 & $(0.041)$ & 2.607 & $(0.048)$ & -0.04 & (13.80) \\
\hline WR 88 & -146.1 & $(6.6)$ & -0.041 & $(0.043)$ & 2.796 & $(0.061)$ & -0.02 & $(20.26)$ \\
\hline WR 92 & -202.5 & $(6.6)$ & 0.014 & $(0.035)$ & 2.677 & $(0.039)$ & -0.02 & (14.97) \\
\hline WR 103 & -264.5 & $(5.7)$ & 0.002 & $(0.022)$ & 2.541 & $(0.032)$ & 0.02 & (18.67) \\
\hline WR 106 (ELO) & -225.9 & $(12.4)$ & 0.018 & $(0.051)$ & 2.658 & $(0.033)$ & 0.00 & $(27.51)$ \\
\hline WR 106 (OMM) & -224.8 & $(5.0)$ & 0.019 & $(0.016)$ & 2.603 & $(0.035)$ & 0.01 & (12.59) \\
\hline WR 119 (ELO) & -205.9 & $(7.7)$ & 0.005 & $(0.048)$ & 2.706 & $(0.043)$ & 0.01 & (27.09) \\
\hline WR 119 (OMM) & -201.2 & $(7.2)$ & -0.017 & $(0.037)$ & 2.672 & $(0.029)$ & -0.01 & $(20.42)$ \\
\hline WR 121 (ELO) & -211.0 & (15.6) & -0.046 & $(0.058)$ & 2.687 & $(0.052)$ & 0.01 & (38.47) \\
\hline WR 121 (OMM) & -206.5 & $(2.8)$ & 0.001 & $(0.045)$ & 2.674 & $(0.035)$ & 0.04 & (22.14) \\
\hline
\end{tabular}


Table 4. Measurements of integrated line quantities.

\begin{tabular}{cccccr}
\hline Star name & $\begin{array}{c}\text { HJD } \\
- \text { HJD(2000) }\end{array}$ & $\begin{array}{c}\text { Equivalent } \\
\text { widths }(\AA)\end{array}$ & Skewness & Kurtosis & \multicolumn{1}{c}{$\begin{array}{c}\text { Radial } \\
\text { velocities } \\
\text { v(km s }\end{array}$} \\
\hline WR 53 & 3426.04 & $-424.3 \pm 6.3$ & $0.024 \pm 0.007$ & $2.299 \pm 0.012$ & $9.9 \pm 2.0$ \\
& 3429.97 & $-432.4 \pm 12.8$ & $0.051 \pm 0.016$ & $2.308 \pm 0.028$ & $-2.5 \pm 4.1$ \\
& 3430.98 & $-418.5 \pm 10.1$ & $0.034 \pm 0.013$ & $2.310 \pm 0.022$ & $-17.9 \pm 3.0$ \\
& & $\ldots$ & & \\
\hline
\end{tabular}

from Table 2. In other words, the change in the global strength of this spectral feature is correlated with the level of variation of the line flux averaged over the width of the line. This indicates that these variations correspond to genuine changes in the line intensity and not only to line flux reorganization in projected velocity space. Note that the presence of CIRs in the wind does not always lead to clear variations in line EW. Chené \& St-Louis (2010), for example, detected a typical S-type pattern characteristic of periodic CIR-type variability in the grey-scale plot of the differences from the mean of the He II $\lambda 5411$ line of WR 1 but no clear period can be identified in the EW curve. On the other hand, for WR 6, another star showing CIR-type variability in the line profile shapes, Morel et al. (1997) found periodic EW variations for many lines in the optical.

The skewness variations range from a standard deviation of 0.01 for WR 53 to 0.058 for WR 121 while the kurtosis values range from a standard deviation of 0.01 for WR 53 to 0.061 for WR 88. No clear periodicity was found in these measurements, which reflect the changes in the shape of the line, except for WR 103 which will be discussed elsewhere. The variations in the RVs calculated using two different methods (bisector and cross-correlation) have amplitudes of $\sim 30-40 \mathrm{~km} \mathrm{~s}^{-1}$ for all stars except WR 53, which displayed a smaller amplitude of variation $\left(\sim 20 \mathrm{~km} \mathrm{~s}^{-1}\right)$. Again, no periodicity was found but a clear anticorrelation was identified for all stars except WR 53 between the RV and skewness changes. We interpret this result as indicating that the changes in RV result from variations in the skewness of the line and not in a real movement of the star.

To illustrate the type of spectral variability we detect, we have plotted in Fig. 10 a montage of the C III $\lambda 5696$ line profiles of WR 69 for the entire ELO campaign. At the left of each profile, we indicate the Julian Date and on the right, the measured skewness (see the online material for the errors on the skewness values). From this figure, it is clear that the numerical values of the measured skewness cannot always easily be associated with the visual appearance of the profile. For example, although the first (HJD-HJD $(2000)=3426.58)$ and third $(\operatorname{HJD}-\mathrm{HJD}(2000)=3430.49)$ profiles both look fairly symmetrical, this is not reflected in their respective value of the skewness, -0.001 , i.e. very close to 0 , and +0.052 . On closer inspection, the lower intensity of the blue edge of the first line profile compared to the mean is probably responsible for the lower skewness value; otherwise, both profiles are almost identical. Two profiles taken $1 \mathrm{~d}$ apart, the sixth (HJD-HJD(2000) $=3433.54)$ and seventh (HJD-HJD(2000) = 3434.51) for example can have skewness values with comparable values but opposite signs $(+0.013$ and -0.016$)$ although they appear to both have an excess emission on the red side of the profile. Therefore the skewness values must be interpreted with care as it is an integrated quantity that reflects the asymmetry of the entire line profile.

\section{DISCUSSION}

As previously found by SLC and CSL, we observe that all WC 9 stars in our sample display LSV ( $\gtrsim 5$ per cent), although despite our higher number of spectra and a more intense observing cadence, we were not able to identify any clear periodicity in the variations. The shape of the $\sigma$ profile characterizing the line variability indicates that the highest levels of changes occur in the line wings which reflects slight changes in the line width and can be qualified as line breathing. For WR 69 for which our data quality is the best, we find that the level of variability for all spectral lines is similar, supporting the suggestion by SLC and CSL that a global effect in the wind is responsible for the variations. The wavelet analysis we performed on the line profiles of our WC 9 stars led us to confirm that the typical mean line-of-sight velocity dispersion of the excess emission peaks is intermediate, $\overline{\sigma_{\xi}} \sim 150-300 \mathrm{~km} \mathrm{~s}^{-1}$, which is slightly larger than that produced by clumps (stars classified as SSV by SLC and CSL) but not as broad as the excess emission bumps found in WN stars identified as LSV and considered as potential CIR candidates. However, we wish to emphasize that because WC 9 stars have narrow lines, the changes are broader in a relative sense, compared to the width of the spectral lines. Finally, our data also support the general trend suggested by CSL that in general higher $\sigma$ values are associated with higher $\overline{\sigma_{\xi}}$ values.

WR 103 is an exception among the WC 9 stars in our sample as it shows lower amplitude changes of $\langle\sigma$ (per cent $)\rangle \sim 4$ per cent and for this star we find a period (of $\sim 9 \mathrm{~d}$ ) in the line profile shapes and line integrated quantities. That star was already known to be somewhat peculiar among WC 9 stars as it shows a higher level of photometric variability (Fahed, Moffat \& Bonanos 2009) despite its lower spectroscopic variability level. A complete analysis of our data on WR 103 will be presented in an upcoming paper.

As for the only WC 8d star in our sample, WR 53, it shows a much lower level of variability $(\sigma=2.20$ per cent) compared to the WC 9 stars. As mentioned by CSL, this demonstrates that LSV is not necessarily a common feature of all dust producers. The shape of its $\sigma$ profile is also different indicating a lower level of variability in the line wings. Interestingly, despite its lower level of variability, we obtain the same $\overline{\sigma_{\xi}}$ value for WR 53 than for our sample of WC 9 stars. In view of the seemingly different nature of the variability for this star compared to the others, it is plausible that a different mechanism is responsible for the formation of dust.

Let us now consider various possible interpretations for the origin of the line profiles changes we observe and examine if these scenarios provide some clues as to the formation mechanism of the dust in the winds of these stars. We note that all the stars in our sample are known to form dust except WR 81, WR 88 and WR 92. In a recent study of the three WC 9 stars WR 88, WR 92 and WR 103, Williams et al. (2015) found increasing abundances of carbon and oxygen in the wind of these three stars. The lower $\mathrm{O}$ abundance is a sign that the star is less evolved and indeed the presence of $\mathrm{N}$ III and $\mathrm{N}$ IV 


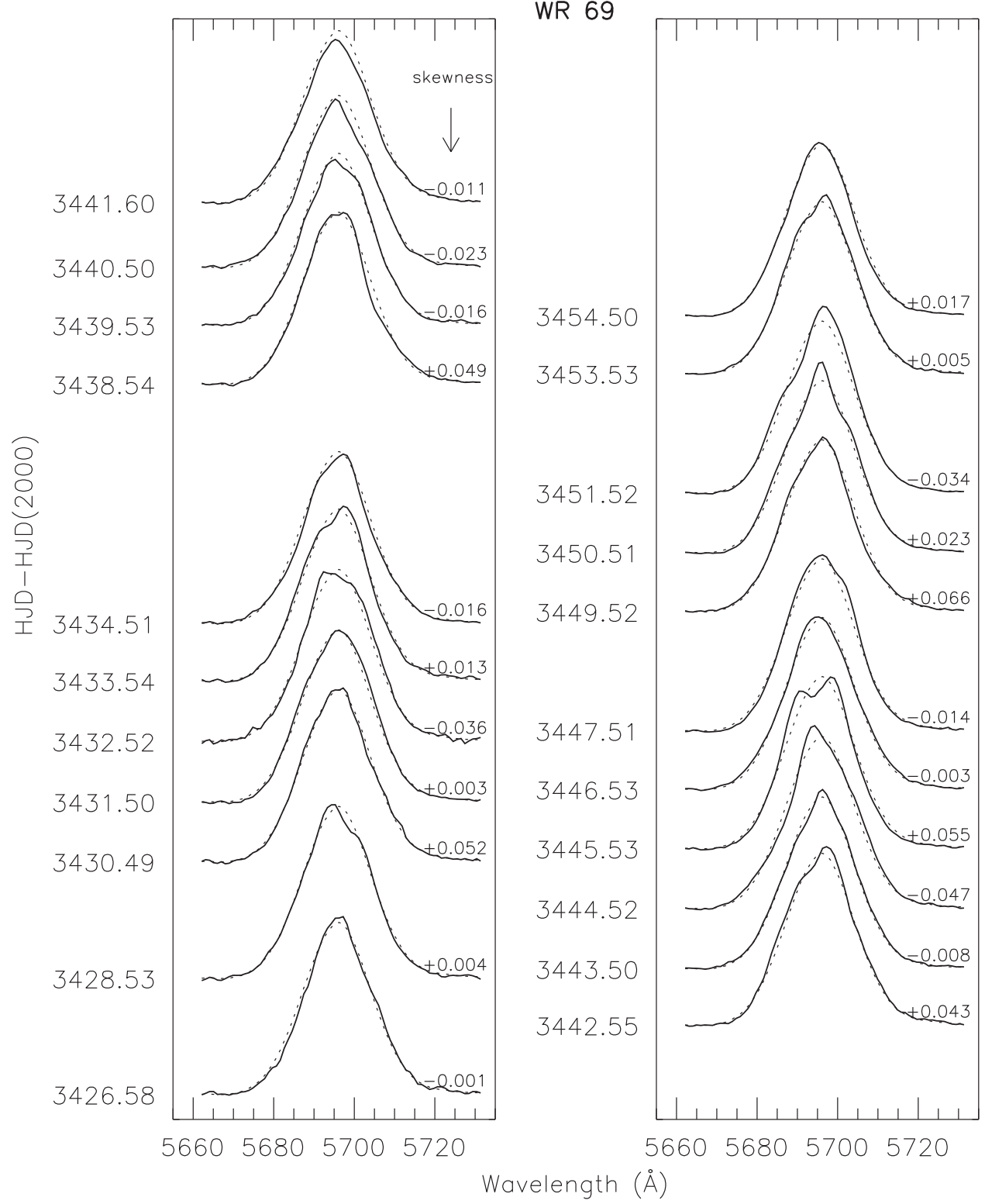

Figure 10. Series of C III $\lambda 5696$ line profiles for WR 69 with the measured skewness value indicated at the bottom right of each profile. See Table 4 for the individual errors on the skewness. The mean profile for the whole series is overploted (dotted line) under each profile for better comparison.

lines in its spectrum led them to re-classify WR 88 as a transitional WN 80/WC 9 star. They suggest that as the star evolves and its $\mathrm{O}$ abundance increases, the accompanying increase in $\mathrm{C}$ abundance is the key element to make it possible for WC 9 stars to form dust in their winds. In a previous study, Williams \& van der Hucht (2000) had also found that WR 81 had anomalously weak OII lines, hinting also to a low $\mathrm{O}$ and possibly $\mathrm{C}$ abundance. Nevertheless, we note that we did not find anything peculiar about the variability pattern of these three non-dust-making WC 9 stars compared to the others.

\subsection{The colliding-wind binary scenario}

Although we have not identified any periodicity for all but one of our WC 9 stars, we have a better idea of the time-scale on which the changes take place. For all our WC 9 stars, the variations occur on a short time-scale, of the order of $1 \mathrm{~d}$ but not hours. Indeed, taking WR 69 as an example, we observe that the profiles can change from being skewed to the blue to being skewed to the red from one day to the next (see Fig. 10). However, for the stars we observed at OMM and for which we have several observations in one night, we have not found a significant large amplitude change of the profile shape within a given night (a few hours of observation). Of course, we do not have sufficiently high $\mathrm{S} / \mathrm{N}$ to observe small-scale changes associated with clumps generally traced by small subpeaks moving from line centre to line edge. These large amplitude line profile shape variations are also not associated with large radial velocity changes. The typical standard deviation we find is around $20 \mathrm{~km} \mathrm{~s}^{-1}$ but does not exceed $40 \mathrm{~km} \mathrm{~s}^{-1}$ and we believe the values can be affected by the fact that the line shape varies as confirmed by the correlation we find between the radial velocity and skewness for all our stars, except perhaps for WR 53. Therefore, if these stars were short-period binaries, they would have relatively low-mass companions with luminosities below the threshold to develop a significant wind and therefore we would not expect a wind-collision shock cone 
to develop and generate the type of changes we observe. However, this does not completely rule out the binary scenario as a source of our line profile changes. Indeed, it is still possible that these WC 9 stars are long- or intermediate-period binaries. In several cases, we have found that observing over a longer time span increases the level of detected line-flux variability indicating that there could be long period changes. Williams \& van der Hucht (2000) have searched for luminous companions for all the stars in our sample except WR 88 using spectra in the blue. They found evidence for absorption lines that can be attributed to a companion only for WR 69. They were not able to assign a formal spectral type, but the presence of $\mathrm{H}$ and He I lines leads them to suggest that the companion cannot be an early $\mathrm{O}$ star. This star is an excellent candidate for follow-up spectroscopic observations on a long time-scale. For the stars in which Williams \& van der Hucht (2000) do not detect absorption lines from a companion, they do not exclude the possibility that there is nevertheless a companion with a sufficiently high luminosity to develop a wind but that it remained undetected in the composite spectrum due to an insufficient $\mathrm{S} / \mathrm{N}$. If the WC 9 stars in our sample are long-period binaries, the variations we observe cannot be explained by the varying line-of-sight projected velocity of the gas in the shock cone as a function of phase. Another mechanism causing changes on a much shorter time-scale has to be identified.

One possible explanation would be instabilities in a non-adiabatic shock cone. In hydrodynamical models of radiatively driven colliding winds, the density, temperature and velocity structures of the colliding-wind shock-cone region depend strongly on the degree of cooling of the gas. Pittard (2009) finds that in the adiabatic case, the structure consists of primary and a secondary shocks separated by a contact discontinuity in which the gas distribution is smooth. However, in the non-adiabatic case, the radiative energy losses cause the shock region to collapse into a thin sheet which is easily perturbed by thin-shell instabilities. As the material flows down the shock cone and crosses the line-formation region of the WR wind, its density and line-of-sight velocity which produce excess emission peaks can change on a time-scale of the flow time along the shock. Lamberts, Fromang \& Dubus (2011) estimate that for binary systems in which the winds collide at unequal speeds, the velocity shear leads to the development of Kelvin-Helmotz instabilities that produce an rms velocity dispersion of some tens of per cent. For systems in which the non-linear thin shell instability dominates, such as for isothermal winds, they find that the amplitude of the velocity perturbations is even larger.

The adiabatic or non-adiabatic nature of the gas in the shock cone depends on the ratio between the cooling time-scale and the dynamical time-scale. In general, the parameter $\chi=v_{8}^{4} d_{12} / \dot{M}_{-7}$ is used to characterize this ratio (Stevens, Blondin \& Pollock 1992). Here $v_{8}$ is the terminal velocity of the pre-shock gas in units of $1000 \mathrm{~km} \mathrm{~s}^{-1}$, $d_{12}$ is the separation between the two stars in units of $10^{12} \mathrm{~cm}$ and $\dot{M}_{-7}$ is the mass-loss rate in units of $10^{-7} \mathrm{M}_{\odot} \mathrm{yr}^{-1}$. If $\chi$ is $\gtrsim 3$, the wind is adiabatic and it is radiative for $\chi \lesssim 3$ (Lamberts et al. 2011). For the WR stars, we can adopt average values of the terminal velocity and mass-loss rate of WC 9 stars from Sander, Hamann \& Todt (2012) of, respectively, $1390 \mathrm{~km} \mathrm{~s}^{-1}$ and $1.6 \times 10^{-5} \mathrm{M}_{\odot} \mathrm{yr}^{-1}$. For the orbital separation, if we assume that the typical radial velocity dispersion we measure, $v_{\mathrm{RV}} \sim 20 \mathrm{~km} \mathrm{~s}^{-1}$, corresponds to the semi-amplitude of the WR star in a circular orbit and assuming a most likely inclination of the orbit with respect to the line of sight of $60^{\circ}$, we can calculate the separation between the two stars as a function of the orbital period. For a 10-d period, we find $d_{12}=$ 0.318 . This gives a very small value of $\chi=0.0077$ and therefore the shock would be non-adiabatic $(\chi<<<1)$. A 2 -yr period leads to $\chi=0.56$, still clearly non-adiabatic. These rough calculations do not include the effects of radiative inhibition (Stevens \& Pollock 1994) that can reduce even more the pre-shock velocities in closer systems and in consequence the value of $\chi$. As for the timescale of the expected changes, if we assume that the velocity of the gas along the shock cone is of the same order of magnitude as the terminal velocity of the wind, we estimate that in $1 \mathrm{~d}$, the gas can easily travel a distance of at least $17 \mathrm{R} \odot$, not incompatible with the typical extent of a line-formation region. We therefore conclude that an origin in unstable gas within a shock cone in an intermediate- to long-period binary is a reasonable interpretation for the line profile changes we observe. The typical velocity width of the structure we detect for WC 9 stars $\left(\sim 150-300 \mathrm{~km} \mathrm{~s}^{-1}\right)$ is roughly compatible with what is expected in unstable colliding-wind shocks (Lamberts et al. 2011) although a hydrodynamical simulation tailored for our particular systems is required for a more detailed comparison.

\subsection{Large- or small-scale inhomogeneities in single-star winds}

Unfortunately, we are forced to conclude that our data set is insufficient to determine if the observed spectroscopic changes occur because of the presence of small- or large-scale inhomogeneities in the wind of a single WR star. To detect subpeak from clumps in the wind moving from line centre to line edge, we need to obtain spectra with a higher $\mathrm{S} / \mathrm{N}$ and observe for a large fraction of a given night as they take several hours to propagate out (e.g. Moffat et al. 1988). There is some evidence that the propagation time is related to the terminal velocity of the wind. For the early WN star WR 2, for example (Chené et al., in preparation), which has a terminal velocity close to $4000 \mathrm{~km} \mathrm{~s}^{-1}$, the subpeaks move out in less than an hour. WC 9 stars have much lower terminal velocities, closer to $1000 \mathrm{~km} \mathrm{~s}^{-1}$. Therefore, we might expect the subpeaks to take much longer to propagate over a detectable projected velocity range. Unfortunately, although we did obtain several spectra per night at OMM, our spectra are typically obtained over a 1-h interval with a maximum of $2 \mathrm{~h}$. In such a short period of time, perhaps it is not surprising that not much change is observed. However, we note that we have found that the typical width of the subpeaks in WC 9 stars seems to be slightly larger than the typical subpeaks clearly associated with clumps in the wind. This is particularly intriguing in view of the small terminal velocities of their winds; the ratio of subpeak width to terminal velocity can approach $1 / 3$ instead of between $1 / 10$ and $1 / 20$ for structures clearly associated with clumps. Only the kinematic behaviour of the subpeaks will be able to provide a definite answer.

As for large-scale structures such as CIRs, in spite of an almost daily monitoring over a period of close to 1 month, we were unable to clearly identify subpeaks moving periodically from one side of the line profile to the other for most of our targets. Although the profiles do not change much in a period of a few hours, as demonstrated by our OMM data, it is still possible that the structures are moving too rapidly on the line profile compared to our sampling for us to detect a periodicity. Indeed, in many cases, we found the line to appear to be skewed to the red to appear being skewed to the blue in $1 \mathrm{~d}$. This would reflect periods shorter than those of WNE stars (a few days) and correspond to higher rotation velocities, particularly in view of the larger radii of these stars. Although the amplitude of the variations is comparable to what is observed in CIR-type variability found in WNE stars, the width of the subpeaks seems to be slightly smaller. This could be related to the lower terminal velocity of the winds of WC 9 stars; the subpeaks appear smaller in absolute terms but not when compared to the line width. 


\section{CONCLUSIONS}

We found that the WC 9 stars in our sample show large amplitude (5-8 per cent) spectroscopic variations with line profile shapes appearing significantly different on time-scales of $1 \mathrm{~d}$. In spite of the short time-scale of the changes, we conclude that in many cases, observing for longer periods of time reveals higher overall values of the percentage of the line flux variability, hinting to process that take place over longer periods of time. The detected subpeaks have widths of $\sim 150-300 \mathrm{~km} \mathrm{~s}^{-1}$, slightly higher than the widths of subpeaks associated with clumps propagating in the wind but smaller in absolute terms (but higher in relative terms when compared to the wind terminal velocity) than those found for CIR-type variations.

With the exception of one star, we did not detect any periodicity in the line profiles changes that might be associated with a binary system or to the presence of CIRs in the wind. The small amplitude of the scatter of the radial velocities of our stars indicates that it is extremely unlikely that these stars are close binary systems. However, intermediate- to long-period binaries cannot be excluded. Furthermore, we conclude that a viable physical process to explain the variability on a short time-scale can be changed in the density and velocity of the gas as it flows along a colliding-wind binary shock cone which is subjected to thin-shell instabilities because of its non-adiabatic nature.

In spite of having observed these stars almost daily for a period of about 1 month, we were not able to find periodic behaviour in the movement of these excess subpeaks. We therefore cannot associate them to the presence of a persistent large-scale structure in the winds of these stars. More intense monitoring, using a telescope in well-separated observing sites, will be required to determine the kinematics of the variable profiles.

\section{ACKNOWLEDGEMENTS}

NS-L acknowledges financial support from the Natural Science and Engineering Research Council (NSERC) of Canada.

\section{REFERENCES}

Chené A.-N., St-Louis N., 2010, ApJ, 716, 929

Chené A.-N., St-Louis N., 2011, ApJ, 736, 140 (CSL)

Cherchneff I., Le Teuff Y. H., Williams P. M., Tielens A. G. G. M., 2000, A\&A, 357, 572

Cranmer S. R., Owocki S. P., 1996, ApJ, 462, 469

Crowther P. A., De Marco O., Barlow M. J., 1998, MNRAS, 296, 367
Fahed R., Moffat A. F. J., Bonanos A. Z., 2009, MNRAS, 392, 376

Fullerton A. W., Gies D. R., Bolton C. T., 1996, ApJS, 103, 475

Lamberts A., Fromang S., Dubus G., 2011, MNRAS, 418, 2618

Lépine S., Moffat A. F. J., 1999, ApJ, 514, 909 (LM)

Michaux Y. J. L., Moffat A. F. J., Chené A.-N., St-Louis N., 2014, MNRAS, 440, 2 (M2014)

Moffat A. F. J., Drissen L., Lamontagne R., Robert C., 1988, ApJ, 334, 1038

Monnier J. D., Tuthill P. G., Danchi W. C., 1999, ApJ, 525, L97

Morel T., St-Louis N., Marchenko S. V., 1997, ApJ, 482, 470

Morel T. et al., 1999, ApJ, 518, 428

Pittard J. M., 2007, ApJ, 660, L141

Pittard J. M., 2009, MNRAS, 396, 1743

Sander A., Hamann W.-R., Todt H., 2012, A\&A, 540, A144

Stevens I. R., Pollock A. M. T., 1994, MNRAS, 269, 226

Stevens I. R., Blondin J. M., Pollock A. M. T., 1992, ApJ, 386, 265

St-Louis N., Chené A.-N., Schnurr O., Nicol M.-H., 2009, ApJ, 698, 1951 (SLC)

Tuthill P. G., Monnier J. D., Danchi W. C., 1999, Nature, 398, 487

Usov V. V., 1991, MNRAS, 252, 49

van der Hucht K. A., 2001, New Astron. Rev., 45, 135

Williams P., 2011, Bull. Soc. R. Sci. Liege, 80, 595

Williams M., van der Hucht K. A., 1992, in Drissen L., Leitherer C., Nota A., eds, ASP Conf. Ser. Vol. 22, Nonisotropic and Variable Outflows from Stars. Astron. Soc. Pac., San Francisco, p. 269

Williams P. M., van der Hucht K. A., 2000, MNRAS, 314, 23

Williams P. M., van der Hucht K. A., The P. S., 1987, QJRAS, 28, 248

Williams P. M., van der Hucht K. A., Rauw G., 2005, in Rauw G., Nazé Y., Blomme R., Gosset E., eds, Massive Stars and High-Energy Emission in OB Associations. A Workshop of the JENAM 2005, Distant Worlds, held in Liège, Belgium, p. 65

Williams P. M., van der Hucht K. A., van Wyk F., Marang F., Whitelock P. A., Bouchet P., Gunawan D. Y. A. S., 2013, MNRAS, 429, 494

Williams P. M., Crowther P. A., van der Hucht K. A., 2015, MNRAS, 449 , 1834

\section{SUPPORTING INFORMATION}

Supplementary data are available at MNRAS online.

\section{mnras_DESFORGES - online.pdf}

Please note: Oxford University Press is not responsible for the content or functionality of any supporting materials supplied by the authors. Any queries (other than missing material) should be directed to the corresponding author for the article.

This paper has been typeset from a $\mathrm{T}_{\mathrm{E}} \mathrm{X} / \mathrm{L} \mathrm{T} \mathrm{E} \mathrm{X}$ file prepared by the author. 